\title{
Successful Management of Ornithine Transcarbamylase Deficiency Presenting with Reversible Metabolic Stroke in a Child
}

๑ Özge Dedeoğlu1, • Çiğdem Kasapkara2, • Kader Karlı Oğuz3, • Esma Altınel4, • Ayşe Aksoy¹

1 University of Health Sciences, Ankara Dr. Sami Ulus Women and Children's Hospital, Clinic of Pediatric Neurology, Ankara, Turkey

2 University of Health Sciences, Ankara Dr. Sami Ulus Women and Children's Hospital, Clinic of Pediatric Metabolism and Nutrition, Ankara, Turkey

3 Hacettepe University Faculty of Medicine, Department of Radiology, Ankara, Turkey

4University of Health Sciences, Ankara Dr. Sami Ulus Women and Children's Hospital, Clinic of Pediatrics, Ankara, Turkey

Keywords: Metabolic stroke, ornithine transcarbamylase deficiency, successful treatment

Dear Editor;

Ornithine transcarbamylase deficiency (OTC) is the most common urea cycle disorder with an estimated prevalence of 1 per 80000 births. In a genetic alteration of ornithine transcarbamylase, enzyme in the hepatic mitochondria leads to the accumulation of ammonia and its metabolites (1). It usually causes hyperammonemic encephalopathy in males during the neonatal to infantile period whereas female carriers present with variable manifestations depending on their pattern of random chromosome $X$ inactivation or spontaneous mutations (2). Hyperammonemia represents a medical emergency requiring prompt treatment to prevent severe neurological damage, coma, and death. We present a rare cause of metabolic stroke due to late onset OTC deficiency that has been successfully managed with a combined therapy of sodium benzoate, arginine and a protein-restricted diet. No neurological damage was apparent at 9 months after treatment. A 6-year-old girl presented with vomiting for 6 months that was accompanied by episodes of anxiety and intermittent irritability. She was born at term with a birth weight of $3.9 \mathrm{~kg}$ to nonconsanguineous healthy parents. Antenatal history and neonatal period were unremarkable. Mental and motor development was normal. On neurological examination, she was anxious, disorientated, markedly agitated, and unable to follow commands. There was no fever, meningism or focal neurological signs. The remainder of the examination was normal. We learned that protein intake was inadequate for her age, that she had been fed only milk for the last week and also 5 months before she had been taken to hospital with the same complaints, getting the diagnosis of probable viral encephalitis, cerebrospinal fluid analysis, neuroimaging and electroencephalogram had been reported as normal. Laboratory investigations including serum glucose, electrolytes, thyroid stimulating hormone, free thyroxine, lactate, pyruvate, erythrocyte sedimentation rate, C-reactive protein, renal and liver function tests were all 

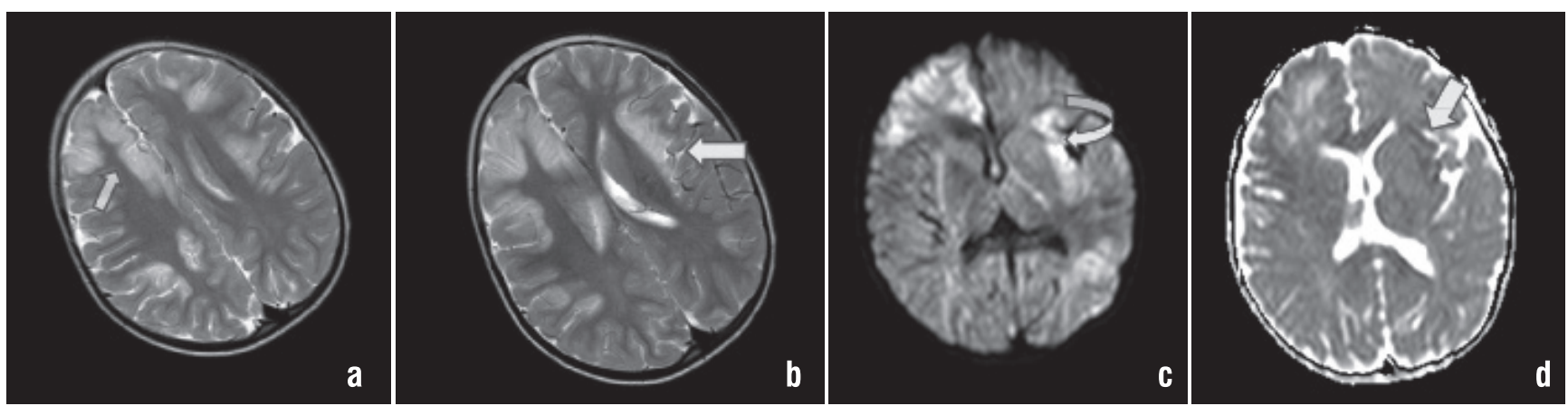

Figure 1. a, b) Axial FLAIR images [TR (repetition time)/TE (echo time); 3000/100 ms)] show bilateral lesions of insulae, frontal lobes and cingulate gyrus. $c, d$ ) images and axial isotropic trace diffusion images (TR/TE; 6600/89 ms with a maximum b value of $1000 \mathrm{~s} / \mathrm{mm}^{2}$ ) reveal restricted diffusion pattern corresponding apparent diffusion coefficient maps reveal low values in the lesions

within reference ranges. The initial ammonia level illustrated $368 \mu \mathrm{mol} / \mathrm{L}$ (reference interval: $16-47 \mu \mathrm{mol} / \mathrm{L})$. Brain magnetic resonance (MR) imaging revealed bilateral lesions of the insulae, frontal lobes and cingulate gyrus on axial T2-weighted images and corresponding apparent diffusion coefficient maps revealed low values in the lesions considering a metabolic stroke (Figure 1). Plasma homocycteine level was $8 \mu \mathrm{mol} / \mathrm{L}$ (reference interval: 5.0 and $15.0 \mu \mathrm{mol} / \mathrm{L}$ ) and the result of cardiac examination was normal. After the exclusion of acquired disorders as the explanation for the hyperammonemia (infections and toxic reasons), the suspicion of a urea cycle defect became stronger in the light of the anamnesis. Metabolic studies obtained before the initiation of therapy were notable for significantly elevated urine orotic acid in organic acid analysis. Blood amino acid analysis conducted simultaneously revealed an increase in glutamine and a decrease in arginine, citrulline. Intravenous dextrose emulsion together with sodium benzoate $(250 \mathrm{mg}$ per kg bodyweight per day), arginine and a protein-restricted diet were started considering OTC deficiency. Within $72 \mathrm{~h}$ she was conscious and feeling better with no permanent sequelae. She was discharged on oral sodium benzoate, phenylbutarate, L-citrulline and arginine. A known nonsense spontaneous mutation c.67C>T (p.Arg23*) was detected in exon 1 at heterozygous state at OTC gene. Her sister and parents were not carriers for the above mentioned mutation found in our patient. Glutamine peak on brain MR spectroscopy supported our diagnosis and the follow-up MR imaging performed 3 months later indicated hyperintense areas in both insulae and frontal lobes on axial T2-weighted images (Figure 2). Her metabolic situation remained stable, and she had no attacks for 9 months, keeping a normal physical and neurological state. Metabolic disorders (urea cycle disorders, branched-chain organic acidurias, mitochondrial diseases) are rare causes of stroke in children (3), which can present with various symptoms including vomiting, lethargy, seizures, coma. The heterogeneity of transience and onset in late-onset OTC deficiency often delay the diagnosis. Several clues such as voluntary protein avoidance and episodic feature of attacks can help to diagnose the condition during the asymptomatic period. Especially in a catabolic state

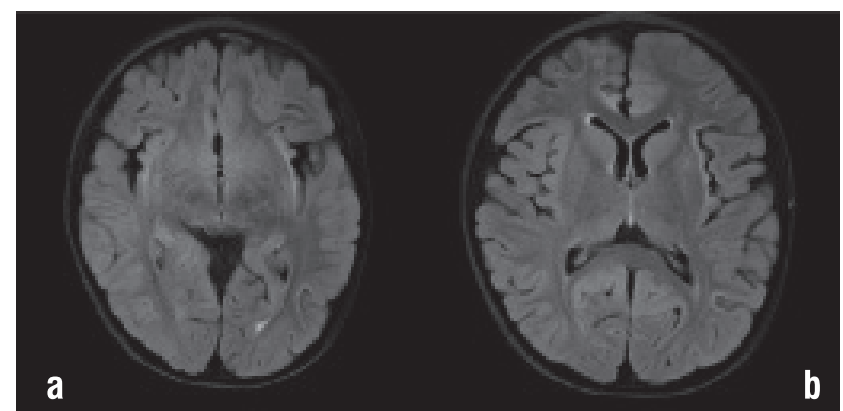

Figure 2. a, b) Axial FLAIR images (repetition time/echo time; 3000/100 $\mathrm{ms}$ ) performed 3 months later indicated hyperintense areas in both insulae and frontal lobes

ammonia levels can rise quickly causing behavioural changes and vomiting, similar to our case (2). Upon a more thorough anamnestic evaluation, our patient's mother reported voluntary protein avoidance of the child. Excessive protein load is a trigger of an attack which is a specific trigger for urea cycle defects. Hyperammonemia and glutamate mediated excitotoxicity have been proposed as the main factor in the pathophysiology of impaired consciousness and seizures but stroke-like episodes in OTC deficiency may or may not be correlated with serum ammonia levels. The formation of glutamine from ammonia and glutamate in cerebral astrocytes leads to increased intracellular osmolarity, cell loss and cytokine release. Cytotoxic cerebral oedema causes impaired cerebral autoregulation and an increase in cerebral blood flow that results in diffusion restricted stroke-like white matter, insular and perirolandic region lesions $(4,5)$. A study which investigated the difference of infarction distribution between the metabolic stroke and non-metabolic stroke group reported that metabolic stroke had no particular influence on anterior circulation or posterior circulation and the most common infarction pattern was deep infarction and border zone infarction (6). Urea cycle defects should be considered in the presence of history and neuroimaging findings. Our patient's brain MR imaging indicated T2 hyperintense lesions of insulae, frontal lobes, cingulate gyrus with restricted diffusion pattern that supported metabolic stroke. Similar to our case a female patient with OTC deficiency had been 
treated with a combined therapy of continuous venovenous hemodialysis and $\mathrm{N}$-carbamylglutamate. Her computed tomography examination performed a few weeks after the coma revealed a moderate hypoperfusion of the cerebral cortex without significant perfusive focal defects (7). Mutation analysis revealed a known nonsense spontaneous mutation c.67C $>\mathrm{T}\left(\mathrm{p} . \mathrm{Arg} 23^{*}\right)$ in exon 1 at heterozygous state at OTC gene in our patient (8). In the literature the nonsense mutation was detected in a 13-year-old Japanese girl who presented with an episode of irritability and vomiting (9). The majority of identified mutations reported for OTC deficiency are amino acid replacements. G-to-A and $\mathrm{C}$-to-T transitions are the most frequent substitutions as in our case. Nonsense spontaneous mutations could be responsible in late onset OTC deficiency whereas one-third of the mutations in X-linked lethal disorders most probably postulated due to de novo events. It is suggested that OTC deficiency is a highly pleomorphic disorder in which other genetic and environmental modifiers play an important role (10-12). Mortality and morbidity rates are high in hyperammonemic encephalopathy in children with OTC deficiency, so aggressive management should be prompt as the prognosis is strongly influenced by the duration of the coma and peak ammonia levels. Long-term management with arginine and L-citrulline supplementation is debated to help to decrease the frequency of attacks and improve physical growth $(13,14)$. Consequently late-onset OTC deficiency should be considered in the differential diagnosis of stroke, which is a rare presentation of a treatable disorder and early diagnosis can prevent a fatal outcome. Dietary habits and the recurrence of attacks could be helpful for the early diagnosis of urea cycle disorders with unusual presentations.

\section{Ethics}

Peer-review: External and internal peer-reviewed.

\section{Authorship Contributions}

Concept: Ö.D., Design: Ö.D., A.A., Data Collection or Processing: Ç.S.K., Ö.D., Analysis or Interpretation: A.A., K.K.O., Literature Search: K.K.O., Ç.S.K., E.A., Writing: Ö.D., A.A.

Conflict of Interests: The authors declared no potential conflicts of interest with respect to the research, authorship and/or publication of this article.

Financial Disclosure: The genetic analyses was tested by Mr. Johannes Häberle, University Children's Hospital Zurich. It was not financially supported.

\section{References}

1. Nakamura K, Kido J, Matsumoto $S$ and et al. Clinical manifestations and growth of patients with urea cycle disorders in Japan. J Hum Genet 2016;61:613-6.

2. Choi JH, Lee BH, Kim JH and et al. Clinical outcomes and the mutation spectrum of the OTC gene in patients with ornithine transcarbamylase deficiency. J Hum Genet 2015;60:501-7.

3. Kalidas K, Behrouz R. Inherited metabolic disorders and cerebral infarction. Expert Rev Neurother 2008;8:1731-41.

4. Braissant $\mathrm{O}, \mathrm{Mc}$ Lin VA, Cudalbu C. Ammonia toxicity to the brain. J Inherit Metab Dis 2013;36:595-612.

5. Gropman A. Brain imaging in urea cycle disorders. Mol Genet Metab 2010;1:20-30.

6. Zhang Z, Xiao M, Ye Zandetal. Noncardiogenic stroke patients with metabolic syndrome have more borderzone infarction and intracranial artery stenosis. J Stroke Cerebrovasc Dis 2015;24:629-34.

7. Tummolo A, Favia V, Bellantuono R and et al. Successful early management of a female patient with a metabolic stroke due to ornithine transcarbamylase deficiency. Pediatr Emerg Care 2013;29:656-8.

8. Grompe M, Caskey CT, Fenwick RG. Improved molecular diagnostics for ornithine transcarbamylase deficiency.Am J Hum Genet 1991;48:212-22.

9. Matsuda I, Tanase S. The ornithine transcarbamylase (OTC) gene: mutations in 50 Japanese families with OTC deficiency.Am J Med Genet 1997;71:378-83.

10. Hata A, Setoyama C, Shimada $K$ and et al. Ornithine transcarbamylase deficiency resulting from a C-to-T substitution in exon 5 of the ornithine transcarbamylase gene. Am J Hum Genet 1989;45:123-7.

11. Caldovic L, Abdikarim I, Narain S and et al. GenotypePhenotype Correlations in Ornithine Transcarbamylase Deficiency: A Mutation Update. J Genet Genomics 2015;42:181-94.

12. McCullough BA, Yudkoff M, Batshaw ML and et al.Genotype spectrum of ornithine transcarbamylase deficiency: correlation with the clinical and biochemical phenotype. Am J Med Genet 2000;93:313-9.

13. Oscarson M, Vassiliou D, Nordenström A. Hereditary metabolic diseases with onset in adulthood. Early and correct treatment of acute symptoms can be life-saving. Lakartidningen 2016;1:113.

14. Bergmann KR, McCabe J, Smith TR and et al. Late onset ornithine transcarbamylase deficiency: treatment and outcome of hyperammonemic crisis. Pediatrics 2014;133:1072-6. 